\title{
AN UPPER BOUND FOR HIGHER ORDER EIGENVALUES OF SYMMETRIC GRAPHS
}

\author{
SHINICHIRO KOBAYASHI
}

\begin{abstract}
In this paper, we derive an upper bound for higher order eigenvalues of the normalized Laplace operator associated with a symmetric finite graph in terms of lower order eigenvalues.
\end{abstract}

\section{INTRODUCTION}

Let $G$ be a connected, finite, simple and undirected graph of $N$ vertices. Let $\Delta$ be the normalized Laplace operator assiciated with $G$. The operator $-\Delta$ is identified with a non-negative definite real symmetric matrix of size $N$. Denote by $\lambda_{0} \leq \lambda_{1} \leq \cdots \leq \lambda_{N-1}$ all eigenvalues of $\Delta$ counted with multiplicity. For any connected graph, we have $\lambda_{0}=0$ and its multiplicity is 1 . All the eigenvalues lie in the interval $[0,2]$. We consider the following question: Are there other contraints on the spectrum $\left\{\lambda_{i}\right\}_{i=0}^{N-1}$ ? In particular, is $\lambda_{k+1}$ controlled by past eigenvalues, $\lambda_{1}, \ldots, \lambda_{k}$ ? This question is a discrete analogue of the so-called Payne-Pólya-Weinberger's inequality. For the Dirichlet eigenvalues $0<\lambda_{1}<\lambda_{2} \leq \lambda_{3} \leq \cdots \uparrow \infty$ of the Laplacian on a bounded domain in the Euclid plane, Payne-Pólya-Weinberger [4,5] proved that

$$
\lambda_{k+1}-\lambda_{k} \leq \frac{2}{k} \sum_{i=1}^{k} \lambda_{i} .
$$

This result is extended to arbitrary dimension by Thompson [6]. Later, Hile and Protter [2] and Yang [7] proved sharper inequalities. In particular, Yang [7] proved that

$$
\sum_{i=1}^{k}\left(\lambda_{k+1}-\lambda_{i}\right)^{2} \leq \frac{4}{n} \sum_{i=1}^{k}\left(\lambda_{k+1}-\lambda_{i}\right) \lambda_{i} .
$$

Date: June 13, 2020.

2010 Mathematics Subject Classification. Primary 35P15, 05C50; Secondary $58 \mathrm{C} 40$.

Key words and phrases. graph Laplacian; higher-order eigenvalue; regular graph; symmetric graph. 
Chung and Oden proposed to study of the discrete analogue of their results. For the Dirichlet eigenvalues $\left\{\lambda_{i}\right\}_{i \geq 1}$ of the normalized Laplacian on a connected finite subgraph in the integer lattice of rank $n$, $\mathrm{Hua}$, Lin and $\mathrm{Su}[3]$ proved that

$$
\sum_{i=1}^{k}\left(\lambda_{k+1}-\lambda_{i}\right)^{2}\left(1-\lambda_{i}\right) \leq \frac{4}{n} \sum_{i=1}^{k}\left(\lambda_{k+1}-\lambda_{i}\right) \lambda_{i} .
$$

How about the case of the Laplacian without boundary conditions? Unlike the case of the Dirichlet boundary condition, 0 is always an eigenvalue. For the eigenvalues $\left\{\lambda_{i}\right\}_{i \geq 0}$ with $\lambda_{0}:=0$ of the Laplacian on a compact Riemannian homogeneous manifold, Cheng and Yang [1] proved that

$$
\sum_{i=0}^{k}\left(\lambda_{k+1}-\lambda_{i}\right)^{2} \leq \sum_{i=1}^{k}\left(\lambda_{k+1}-\lambda_{i}\right)\left(4 \lambda_{i}+\lambda_{1}\right) .
$$

In this paper, we consider a discrete analogue of (1.2). More precisely, for a finite symmetric graph, we prove a discrete analogue of (1.2).

Theorem 1.1. Let $G$ be an symmetric finite graph with $N$ vertices. Denote by $0=\lambda_{0}<\lambda_{1} \leq \lambda_{2} \leq \cdots \leq \lambda_{N-1}$ the all eigenvalues of the normalized Laplace operator. Then, for any non-zero eigenvalue $\lambda$ of $\Delta$, we have

$$
\sum_{i=0}^{k}\left(\lambda_{k+1}-\lambda_{i}\right)^{2}\left(1-\lambda_{i}\right) \leq \sum_{i=0}^{k}\left(\lambda_{k+1}-\lambda_{i}\right)\left(2(2-\lambda) \lambda_{i}+\lambda\right) .
$$

By using Chebyshev's sum inequality, we obtain an upper bound of $\lambda_{k+1}$ in terms of $\lambda_{1}, \ldots, \lambda_{k}$.

Theorem 1.2. In the same setting as Theorem 1.1, we have

$$
\lambda_{k+1} \leq \frac{(k+1) \lambda_{1}+\sum_{i=1}^{k}\left(\left(5-2 \lambda_{1}\right) \lambda_{i}-\lambda_{i}^{2}\right)}{\sum_{i=0}^{k}\left(1-\lambda_{i}\right)} .
$$

Let $\mu_{1}:=\lambda_{1}$ and $m$ be the multiplicity of $\mu_{1}$. If $G$ is not a complete graph, then we can consider $\mu_{2}:=\lambda_{m+1}$, i.e., the second smallest positive eigenvalue. We have a upper bound for the ratio $\mu_{2} / \mu_{1}$ in terms of the multiplicity of $\mu_{1}$.

Corollary 1.3. In the same setting as Theorem 1.1, let $m$ be the multiplicity of $\mu_{1}$ and put $\mu_{2}:=\lambda_{m+1}$. Then, we have

$$
\frac{\mu_{2}}{\mu_{1}} \leq 3 m+1
$$

Acknowledgment. The author would like to thank Professor Takashi Shioya for helpful comments. 


\section{Preliminaries}

In this section, unless otherwise stated, we assume that all graphs are connected, finite, simple and undirected. We recall some basic facts on the theory of eigenvalues of a regular graph. Let $G=(V, E)$ be a $d$-regular graph, $d \geq 1$, and put $N:=\# V$. If two vertices $x, y \in V$ are adjacent, then we denote this situation by $x \sim y$. Note that since $G$ is undirected, $x \sim y$ if and only if $y \sim x$. The normalized Laplace operator $\Delta$ acting on the space $C(V)$ of functions on $V$ is defined by

$$
\Delta u(x):=\frac{1}{d} \sum_{y \sim x}(u(y)-u(x)), u \in C(V), x \in V .
$$

The normalized Laplace operator is identified with the real-symmetric matrix $D^{-1} A-I$, where $D$ is the scalar matrix with diagonal entries $d$, $A$ is the adjacency matrix of $G$ and $I$ is the identity matrix. A complex number $\lambda$ is called an eigenvalue of $\Delta$ if there exists $u \in C(V) \backslash\{0\}$ such that $\Delta u+\lambda u=0$ holds. In this case, the function $u$ is called an eigenfunction with eigenvalue $\lambda$. For an eigenvalue $\lambda$ of $\Delta$, we denote by $W_{\lambda}$ the space of all functions $u \in C(V)$ satisfying $\Delta u+\lambda u=0$ and we call the dimension of $W_{\lambda}$ multiplicity of $\lambda$. Let us denote the eigenvalues of $\Delta$ by $\lambda_{0} \leq \lambda_{1} \leq \lambda_{2} \leq \cdots \leq \lambda_{N-1}$, counted with multiplicity. We define a inner product $\langle\cdot, \cdot\rangle$ on $C(V)$ by

$$
\langle u, v\rangle:=\sum_{x \in V} u(x) v(x) d
$$

We denote by $\|\cdot\|$ the norm induced by the inner product $\langle\cdot, \cdot\rangle$. We list up some elementary facts on eigenvalues and eigenfunctions without proofs.

- 0 is an eigenvalue of multiplicity 1 and constant functions are eigenfunctions with eigenvalue 0 .

- All eigenvalues lie in the interval $[0,2] \subset \mathbb{R}$.

- There exists a orthonormal basis $\left\{u_{i}\right\}_{i=0}^{N-1}$ of $C(V)$ such that each function $u_{i}$ is an eigenfunction with eigenvalue $\lambda_{i}$.

By the min-max formula, each eigenvalue $\lambda_{k}$ has a variational characterization:

$$
\lambda_{k}=\inf \left\{\frac{\sum_{x \sim y}(u(y)-u(x))^{2}}{2 d \sum_{V} u^{2}} \mid \begin{array}{l}
u \neq 0,\left\langle u, u_{i}\right\rangle=0, i=0, \ldots, k-1 \\
3
\end{array}\right\}
$$


where the symbol $\sum_{x \sim y}$ means the summation over all unordered pairs $(x, y)$ such that $x \sim y$. In particular, we have

$$
\lambda_{1}=\inf \left\{\frac{\sum_{x \sim y}(u(y)-u(x))^{2}}{2 d \sum_{V} u^{2}} \mid u \neq 0, \sum_{V} u=0\right\} .
$$

We shall derive a general upper bound for $\lambda_{1}$.

Lemma 2.1. For any regular graph $G$ but a complete graph, we have

$$
\lambda_{1} \leq 1
$$

Proof. Since $G$ is not complete, there exist two vertices $x_{0}, y_{0} \in V$ such that $x_{0} \nsim y_{0}$. We define a function $u \in C(V)$ by

$$
u(x):= \begin{cases}1 & \text { if } x=x_{0} \\ -1 & \text { if } x=y_{0} \\ 0 & \text { otherwise. }\end{cases}
$$

Clearly, the function $u$ satisfies $\sum_{V} u=0$. From (2.1), we have

$$
\lambda_{1} \leq \frac{\sum_{x \sim y}(u(y)-u(x))^{2}}{2 d \sum_{V} u^{2}}=1
$$

Remark 2.2. If $G$ is the complete graph of degree $d$, then $\lambda_{1}=1+1 / d$.

Let $\Gamma: C(V) \times C(V) \rightarrow C(V)$ be the carré du champ operator associated to $\Delta$, i.e., for $u, v \in C(V)$,

$$
\Gamma(u, v):=\frac{1}{2}(\Delta(u v)-(\Delta u) v-u \Delta v) .
$$

For two vertices $x, y \in V$ with $x \sim y$, we define the difference operator $\nabla_{x y}: C(V) \rightarrow C(V)$ by

$$
\nabla_{x y} u:=u(y)-u(x), u \in C(V) .
$$

By a simple calculation, we have

$$
\Gamma(u, v)(x)=\frac{1}{2 d} \sum_{y \sim x}\left(\nabla_{x y} u\right)\left(\nabla_{x y} v\right), x \in V .
$$

The carré du champ $\Gamma(u, v)$ is an analogy of $\langle\nabla u, \nabla v\rangle$ in the context of Riemannian geometry, where $\nabla$ is the gradient operator. We list up some identities for $\Gamma$.

Lemma 2.3. Let $u, v, v_{1}, v_{2} \in C(V)$.

(1) $\langle u, \Delta v\rangle=-\sum_{V} \Gamma(u, v) d$. 
(2) For any $x \in V$, we have

$$
\begin{aligned}
\Gamma\left(u, v_{1} v_{2}\right)(x)=\Gamma\left(u, v_{1}\right) & v_{2}(x)+\Gamma\left(u, v_{2}\right) v_{1}(x) \\
& +\frac{1}{2 d} \sum_{y \sim x}\left(\nabla_{x y} u\right)\left(\nabla_{x y} v_{1}\right)\left(\nabla_{x y} v_{2}\right) .
\end{aligned}
$$

In particular,

$$
\sum_{V} \Gamma\left(u, v_{1} v_{2}\right)=\sum_{V}\left(\Gamma\left(u, v_{1}\right) v_{2}+\Gamma\left(u, v_{2}\right) v_{1}\right) .
$$

Making use of the min-max formula and appropriate trial functions, we have the following lemma.

Lemma 2.4. Let $k \geq 1$ be an integer. For any function $h \in C(V)$, we have

$$
\frac{1}{2} \sum_{i=0}^{k}\left(\lambda_{k+1}-\lambda_{i}\right)^{2} \Phi_{i}(h) \leq \sum_{i=0}^{k}\left(\lambda_{k+1}-\lambda_{i}\right)\left\|2 \Gamma\left(h, u_{i}\right)+u_{i} \Delta h\right\|^{2},
$$

where $\Phi_{i}(h)=\sum_{x \sim y} u_{i}(x) u_{i}(y)\left(\nabla_{x y} h\right)^{2}$.

Proof. Let $h \in C(V)$. For $i=0, \ldots, k$, define $\varphi_{i} \in C(V)$ as the orthogonal projection of $h u_{i}$ to the subspace spanned by $\left\{u_{k+1}, \ldots, u_{N-1}\right\}$, i.e.,

$$
\varphi_{i}:=h u_{i}-\sum_{j=0}^{k} a_{i j} u_{j}, a_{i j}:=\left\langle h u_{i}, u_{j}\right\rangle .
$$

Clearly the function $\varphi_{i}$ is perpendicular to $u_{0}, \ldots, u_{k}$. The min-max formula yields

$$
\lambda_{k+1}\left\|\varphi_{i}\right\|^{2} \leq \frac{1}{2} \sum_{x \sim y}\left(\nabla_{x y} \varphi_{i}\right)^{2}=\sum_{V} \Gamma\left(\varphi_{i}, \varphi_{i}\right) d .
$$

From (1) in Lemma 2.3 and the fact that $\left\langle\varphi_{i}, u_{j}\right\rangle=0$ for $j=0, \ldots, k$, we have

$$
\begin{aligned}
\sum_{V} \Gamma\left(\varphi_{i}, \varphi_{i}\right) d & =-\left\langle\varphi_{i}, \Delta \varphi_{i}\right\rangle \\
& =-\left\langle\varphi_{i}, 2 \Gamma\left(h, u_{i}\right)+u_{i} \Delta h-\lambda_{i} u_{i} h+\sum_{j=0}^{k} a_{i j} \lambda_{j} u_{j}\right\rangle \\
& =-\left\langle\varphi_{i}, 2 \Gamma\left(h, u_{i}\right)+u_{i} \Delta h-\lambda_{i} u_{i} h\right\rangle \\
& =-\left\langle\varphi_{i}, 2 \Gamma\left(h, u_{i}\right)+u_{i} \Delta h\right\rangle+\lambda_{i}\left\|\varphi_{i}\right\|^{2} .
\end{aligned}
$$

From (2.2), we obtain

$$
\left.\left(\lambda_{k+1}-\lambda_{i}\right)\left\|\varphi_{i}\right\|^{2} \leq \underset{5}{-\left\langle\varphi_{i}\right.}, 2 \Gamma\left(h, u_{i}\right)+u_{i} \Delta h\right\rangle .
$$


Let $A_{i}$ be the right hand side of 2.3 . We estimate $A_{i}$ in two ways. First, we claim that

$$
A_{i}=\frac{1}{2} \sum_{x \sim y} u_{i}(x) u_{i}(y)\left(\nabla_{x y} h\right)^{2}+\sum_{j=0}^{k}\left(\lambda_{i}-\lambda_{j}\right) a_{i j}^{2} .
$$

To see (2.4), we use Lemma 2.3. By the definition of $\varphi_{i}$,

$$
A_{i}=\sum_{j=0}^{k} a_{i j}\left\langle u_{j}, u_{i} \Delta h+2 \Gamma\left(h, u_{i}\right)\right\rangle-d \sum_{V}\left(h u_{i}^{2} \Delta h+2 h u_{i} \Gamma\left(h, u_{i}\right)\right) .
$$

The first term is equal to $\sum_{j=0}^{k}\left(\lambda_{i}-\lambda_{j}\right) a_{i j}^{2}$. Indeed, by the definition of $\Gamma\left(h, u_{i}\right)$ and Lemma 2.3 , we have

$$
\begin{aligned}
\left\langle u_{j}, u_{i} \Delta h+2 \Gamma\left(h, u_{i}\right)\right\rangle & =\left\langle u_{j}, \Delta\left(h u_{i}\right)+\lambda_{i} h u_{i}\right\rangle \\
& =\lambda_{i} a_{i j}-\left\langle\lambda_{j} u_{j}, h u_{i}\right\rangle \\
& =\left(\lambda_{i}-\lambda_{j}\right) a_{i j} .
\end{aligned}
$$

The second term is equal to $\sum_{x \sim y} u_{i}(x) u_{i}(y)\left(\nabla_{x y} h\right)^{2} / 2$. Indeed,

$$
\begin{aligned}
-\left\langle h u_{i}^{2}, \Delta h\right\rangle & =\sum_{V} \Gamma\left(h u_{i}^{2}, h\right) d \\
& =\sum_{V}\left(h \Gamma\left(u_{i}^{2}, h\right)+u_{i}^{2} \Gamma(h, h)\right) d \\
& =\frac{1}{2} \sum_{x \sim y}\left(\left(\nabla_{x y} u_{i}\right)^{2} h(x)\left(\nabla_{x y} h\right)+u_{i}(x)^{2}\left(\nabla_{x y} h\right)^{2}\right) \\
& +\sum_{V} 2 h u_{i} \Gamma\left(h, u_{i}\right) d \\
& =\frac{1}{2} \sum_{x \sim y} u_{i}(x) u_{i}(y)\left(\nabla_{x y} h\right)^{2}+\sum_{V} 2 h u_{i} \Gamma\left(h, u_{i}\right) d .
\end{aligned}
$$

Second, we claim that

$$
\left(\lambda_{k+1}-\lambda_{i}\right) A_{i} \leq\left\|u_{i} \Delta h+2 \Gamma\left(u_{i}, h\right)\right\|^{2}-\sum_{j=0}^{k}\left(\lambda_{i}-\lambda_{j}\right)^{2} a_{i j}^{2} .
$$

From the definition of $A_{i}$, we have

$$
A_{i}=-\left\langle\varphi_{i}, 2 \Gamma\left(h, u_{i}\right)+u_{i} \Delta h-\sum_{j=0}^{k}\left(\lambda_{i}-\lambda_{j}\right) a_{i j} u_{j}\right\rangle
$$


Applying the Cauchy-Schwartz inequality to the definition of $A_{i}$ and taking account into (2.3) and (2.5), we have

$$
\left(\lambda_{k+1}-\lambda_{i}\right) A_{i}^{2} \leq A_{i}\left(\left\|2 \Gamma\left(h, u_{i}\right)+u_{i} \Delta h\right\|^{2}-\sum_{j=0}^{k}\left(\lambda_{i}-\lambda_{j}\right)^{2} a_{i j}^{2}\right) .
$$

From (2.4) and (2.6), we obtain

$$
\begin{aligned}
& \frac{1}{2} \sum_{i=0}^{k}\left(\lambda_{k+1}-\lambda_{i}\right)^{2} \sum_{x \sim y} u_{i}(x) u_{i}(y)\left(\nabla_{x y} h\right)^{2}+\sum_{i, j=0}^{k}\left(\lambda_{k+1}-\lambda_{i}\right)^{2}\left(\lambda_{i}-\lambda_{j}\right) a_{i j}^{2} \\
& \leq \sum_{i=0}^{k}\left(\lambda_{k+1}-\lambda_{i}\right)\left\|2 \Gamma\left(h, u_{i}\right)+u_{i} \Delta h\right\|^{2}-\sum_{i, j=0}^{k}\left(\lambda_{k+1}-\lambda_{i}\right)\left(\lambda_{i}-\lambda_{j}\right)^{2} a_{i j}^{2} .
\end{aligned}
$$

Since $\sum_{i, j=0}^{k}\left(\lambda_{k+1}-\lambda_{i}\right)^{2}\left(\lambda_{i}-\lambda_{j}\right) a_{i j}^{2}=-\sum_{i, j=0}^{k}\left(\lambda_{k+1}-\lambda_{i}\right)\left(\lambda_{i}-\lambda_{j}\right)^{2} a_{i j}^{2}$, we complete the proof.

\section{PROOF OF MAIN THEOREM}

In this section, we give a proof of Theorem 1.1. In order to complete the proof, we use some symmetries of eigenfunctions on a symmetric graph.

3.1. Symmetries of eigenfunctions on a symmetric graph. We derive some properties of eigenfunctions on a symmetric graph. In particular, Lemma 3.2 is peculiar to symmetric graphs. A graph $G=$ $(V, E)$ is said to be symmetric if for any two edges $(x, y),\left(x^{\prime}, y^{\prime}\right) \in E$, there exists an automorphism $\gamma$ of $G$ such that $x^{\prime}=\gamma x$ and $y^{\prime}=$ $\gamma y$ hold. We denote by $\operatorname{Aut}(G)$ the group of automorphisms of $G$. Note that symmetric graphs are vertex-transitive, i.e., $\operatorname{Aut}(G)$ acts transitively on $V$, and thus regular. We say that a vector subspace $W$ of $C(V)$ is invariant if for any $u \in W$ and $\gamma \in \operatorname{Aut}(G), \gamma u \in W$, where $\gamma u$ is defined by $\gamma u(x):=u(\gamma x), x \in V$.

Lemma 3.1. Let $G=(V, E)$ be a vertex-transitive graph. Let $W$ be an invariant vector subspace of $C(V)$ of dimension $m$ and let $\left\{u_{\alpha}\right\}_{\alpha=1}^{m}$ be an orthonormal basis of $W$. Then, the function $\left|u_{1}\right|^{2}+\cdots+\left|u_{m}\right|^{2}$ is constant and its value is $m / d \# V$.

Proof. Put $f(x):=\left|u_{1}(x)\right|^{2}+\cdots+\left|u_{m}(x)\right|^{2}$. By the invariance of $W$, the family $\left\{\gamma u_{\alpha}\right\}_{\alpha=1}^{m}$ is also an orthonormal basis of $W$ for any $\gamma \in \operatorname{Aut}(G)$. For fixed $x \in V$, it is easy to see that the sum $\left|u_{1}(x)\right|^{2}+\cdots+\left|u_{m}(x)\right|^{2}$ 
is independent of the choice of an orthonormal basis $\left\{u_{\alpha}\right\}$. Thus,

$$
f(\gamma x)=\sum_{\alpha=1}^{m}\left|\gamma u_{\alpha}(x)\right|^{2}=\sum_{\alpha=1}^{m}\left|u_{\alpha}(x)\right|^{2}=f(x) .
$$

The transitivity of the action of $\operatorname{Aut}(G)$ yields that $f$ is constant. Let $C$ be the value of $\left|u_{1}(x)\right|^{2}+\cdots+\left|u_{m}(x)\right|^{2}$. By multiplying $d$ and summing over $x \in V$, we have

$$
C d \# V=\sum_{\alpha=1}^{m} \sum_{x \in V}\left|u_{\alpha}(x)\right|^{2} d=m .
$$

Lemma 3.2. Let $G$ be a symmetric graph. Let $\lambda$ be an eigenvalue of $\Delta$ and let $\left\{u_{\alpha}\right\}_{\alpha=1}^{m}$ be an orthonormal basis of $W_{\lambda}$. Then, the function $g(x, y):=\sum_{\alpha=1}^{m}\left|\nabla_{x y} u_{\alpha}\right|^{2}, x \sim y$, is constant and its value is $m \lambda / \# E$.

Proof. Since $W_{\lambda}$ is an invariant vector subspace of $C(V)$, the family $\left\{\gamma u_{\alpha}\right\}_{\alpha=1}^{m}$ is also an orthonormal basis of $W_{\lambda}$ for any $\gamma \in \operatorname{Aut}(G)$. Since the sum $\sum_{\alpha=1}^{m}\left|\nabla_{x y} u_{\alpha}\right|^{2}$ is independent of the choice of an orthonormal basis $\left\{u_{\alpha}\right\}$, we have

$$
g(\gamma x, \gamma y)=\sum_{\alpha=1}^{m}\left|\nabla_{x y}\left(\gamma u_{\alpha}\right)\right|^{2}=\sum_{\alpha=1}^{m}\left|\nabla_{x y} u_{\alpha}\right|^{2}=g(x, y) .
$$

The symmetry of $G$ yields that $g$ is constant. Let $C^{\prime}$ be the value of $g$. By summing over $x \sim y$, we have

$$
2 C^{\prime} \# E=\sum_{\alpha=1}^{m} \sum_{x \sim y}\left|\nabla_{x y} u_{\alpha}\right|^{2}=2 \lambda m .
$$

Corollary 3.3. Let $G$ be a symmetric graph. Let $\lambda$ and $\left\{u_{\alpha}\right\}$ be as in Lemma 3.2. Then, the function $f_{3}(x, y)=\sum_{\alpha=1}^{m} u_{\alpha}(x) \nabla_{x y} u_{\alpha}$ is constant and its value is $-\lambda m / 2 \# E$.

Proof. The constancy of $f_{3}$ immediately follows from Lemma 3.1 and Lemma 3.2. Let $C_{3}$ be the value of $f_{3}$. By summing over $x \sim y$, we have

$$
2 C_{3} \# E=\sum_{\alpha=1}^{m} \sum_{x \sim y} u_{\alpha}(x) \nabla_{x y} u_{\alpha} .
$$

By interchanging $x$ and $y$,

$$
\sum_{x \sim y} u_{\alpha}(x) \nabla_{x y} u_{\alpha}=-\sum_{x \sim y} u_{\alpha}(y) \nabla_{x y} u_{\alpha}
$$


Thus, we obtain

$$
2 C_{3} \# E=\frac{1}{2} \sum_{\alpha=1}^{m} \sum_{x \sim y}\left(u_{\alpha}(x)-u_{\alpha}(y)\right) \nabla_{x y} u_{\alpha}=-\lambda m .
$$

3.2. Proof of main theorem. We prove Theorem 1.1, Theorem 1.2 and Corollary 1.3 . First, we prove Theorem 1.1 .

Proof of Theorem 1.1. Let $\left\{u_{\alpha}\right\}_{\alpha}$ be an orthonormal basis of $E_{\mu}$. Then, we have

$$
\begin{aligned}
\sum_{\alpha=1}^{m} \sum_{x \sim y} u_{i}(x) u_{i}(y)\left|\nabla_{x y} u_{\alpha}\right|^{2} & =\frac{\lambda m}{\# E} \sum_{x \sim y} u_{i}(x) u_{i}(y) \\
& =\frac{\lambda m}{\# E} \sum_{x \in V} u_{i}(x) d \cdot \frac{1}{d} \sum_{y \sim x} u_{i}(y) \\
& =\frac{\lambda m}{\# E}\left(1-\lambda_{i}\right) \sum_{x \in V} u_{i}(x)^{2} d \\
& =\frac{\lambda m}{\# E}\left(1-\lambda_{i}\right) .
\end{aligned}
$$

Next, we evaluate $\sum_{\alpha}\left\|2 \Gamma\left(u_{\alpha}, u_{i}\right)+u_{i} \Delta u_{\alpha}\right\|^{2}$. By Jensen's inequality, we have

$$
4 \Gamma\left(u_{i}, u_{\alpha}\right)(x)^{2}=\left(\frac{1}{d} \sum_{y \sim x}\left(\nabla_{x y} u_{i}\right)\left(\nabla_{x y} u_{\alpha}\right)\right)^{2} \leq \frac{1}{d} \sum_{y \sim x}\left(\nabla_{x y} u_{i}\right)^{2}\left(\nabla_{x y} u_{\alpha}\right)^{2},
$$

which yields

$$
4 \sum_{\alpha=1}^{m} \sum_{x \in V} \Gamma\left(u_{i}, u_{\alpha}\right)(x)^{2} d \leq \frac{2 \lambda \lambda_{i} m}{\# E} .
$$

By Lemma 3.1, we have

$$
\sum_{\alpha=1}^{m} \sum_{x \in V}\left(u_{i}(x) \Delta u_{\alpha}(x)\right)^{2} d=\frac{\lambda^{2} m}{2 \# E} .
$$

By Lemma 3.3 ,

$$
\begin{aligned}
-4 \lambda \sum_{\alpha=1}^{m} \sum_{x \in V} u_{i}(x) u_{\alpha}(x) \Gamma\left(u_{\alpha}, u_{i}\right)(x) d & =\frac{\lambda^{2} m}{\# E} \sum_{x \in V} u_{i}(x) \sum_{y \sim x} \nabla_{x y} u_{i} \\
& =-\frac{\lambda^{2} \lambda_{i} m}{\# E}
\end{aligned}
$$


By letting $h=u_{\alpha}$ in Lemma 2.4, summing over $\alpha=1, \ldots, m$ and taking account into (3.1), (3.2), (3.3) and (3.4), we obtain

$$
\sum_{i=0}^{k}\left(\lambda_{k+1}-\lambda_{i}\right)^{2}\left(1-\lambda_{i}\right) \leq \sum_{i=0}^{k}\left(\lambda_{k+1}-\lambda_{i}\right)\left(2(2-\lambda) \lambda_{i}+\lambda\right) .
$$

In order to prove Theorem 1.2 , we need some lemmas.

Lemma 3.4 (Chebyshev's sum inequality). Let $N \geq 1$ be an integer and $\left\{a_{i}\right\}_{i=1}^{N},\left\{b_{i}\right\}_{i=1}^{N}$ two sequences of real numbers. If both of $\left\{a_{i}\right\}_{i=1}^{N},\left\{b_{i}\right\}_{i=1}^{N}$ are non-increasing, then

$$
\frac{1}{N} \sum_{i=1}^{N} a_{i} b_{i} \geq\left(\frac{1}{N} \sum_{i=1}^{N} a_{i}\right)\left(\frac{1}{N} \sum_{i=1}^{N} b_{i}\right) .
$$

Lemma 3.5. For any $0 \leq k \leq N-1$,

$$
\sum_{i=0}^{k}\left(1-\lambda_{i}\right) \geq 0
$$

and the equality holds if and only if $k=N-1$.

Proof. Let $A$ be the adjacency matrix of $G$ and $\nu_{0} \geq \nu_{1} \geq \cdots \geq \nu_{N-1}$ be all eigenvalues of $A$. Since any diagonal entry of $A$ is $0, \sum_{i=0}^{N-1} \nu_{i}$ is also 0 and $\sum_{i=0}^{k} \nu_{i} \geq 0$ for any $k$, with the equality holds if and only if $k=N-1$. By the relation between $\Delta$ and $A$, we have

$$
\sum_{i=0}^{k}\left(1-\lambda_{i}\right)=\frac{1}{d} \sum_{i=0}^{k} \nu_{i} \geq 0
$$

and equality holds if and only if $k=N-1$.

Next, we prove Theorem 1.2 and Corollary 1.3 .

Proof of Theorem 1.2. By letting $\lambda=\lambda_{1}$ in Theorem 1.1, we have

$$
\sum_{i=0}^{k}\left(\lambda_{k+1}-\lambda_{i}\right)\left(\lambda_{i}^{2}-\left(\lambda_{k+1}-2 \lambda_{1}+5\right) \lambda_{i}+\lambda_{k+1}-\lambda\right) \leq 0 .
$$

Clearly, $\lambda_{k+1}-\lambda_{i}$ is non-increasing in $i$. Put $f(x):=x^{2}-\left(\lambda_{k+1}-2 \lambda_{1}+\right.$ $5) x$. Then, the function $f$ is non-increasing in the interval $\left(-\infty,\left(\lambda_{k+1}-\right.\right.$ $\left.2 \lambda_{1}+5\right) / 2$. From Lemma 2.1. $\left(\lambda_{k+1}-2 \lambda_{1}+5\right) / 2 \geq 2$. Since $0 \leq \lambda_{i} \leq 2$, 
$\lambda_{i}^{2}-\left(\lambda_{k+1}-2 \lambda_{1}+5\right) \lambda_{i}+\lambda_{k+1}-\lambda$ is non-increasing in $i$. We may use Lemma 3.4 and thus

$$
\left(\lambda_{k+1}-\sum_{i=0}^{k} \frac{\lambda_{i}}{k+1}\right)\left(\sum_{i=0}^{k} \frac{\left(1-\lambda_{i}\right) \lambda_{k+1}+\lambda_{i}^{2}-\left(5-2 \lambda_{1}\right) \lambda_{i}}{k+1}-\lambda_{1}\right) \leq 0 .
$$

If $k \geq m\left(\lambda_{1}\right)$, then $\lambda_{k+1}-\sum_{i=0}^{k} \lambda_{i} /(k+1)$ is strictly positive. In this case, we have

$$
\frac{1}{k+1} \sum_{i=0}^{k}\left(\left(1-\lambda_{i}\right) \lambda_{k+1}+\lambda_{i}^{2}-\left(5-2 \lambda_{1}\right) \lambda_{i}-\lambda_{1}\right) \leq 0 .
$$

By Lemma 3.5, we obtain

$$
\lambda_{k+1} \leq \frac{(k+1) \lambda_{1}+\sum_{i=1}^{k}\left(\left(5-2 \lambda_{1}\right) \lambda_{i}-\lambda_{i}^{2}\right)}{\sum_{i=0}^{k}\left(1-\lambda_{i}\right)} .
$$

This inequality also holds for $k<m\left(\lambda_{1}\right)$.

Proof of Corollary 1.3. If $k=m\left(\lambda_{1}\right)$, then $\lambda_{k+1}=\mu_{2}$ and $\lambda_{1}=\cdots=$ $\lambda_{k-1}=\mu_{1}$. By Theorem 1.2, we have

$$
\frac{\mu_{2}}{\mu_{1}} \leq \frac{6 m+1-3 m \mu_{1}}{m+1-m \mu_{1}} .
$$

Let $g(x):=(6 m+1-3 m x) /(m+1-m x)$. The function $g$ is increasing. By Lemma 2.1.

$$
\frac{\mu_{2}}{\mu_{1}} \leq g(1)=3 m+1
$$

\section{On the NON-TRIViality of Corollary 1.3}

In this section, we consider symmetric graphs, other than complete graphs. Let $\mu_{1}$ and $\mu_{2}$ be the first and the second smallest positive eigenvalue, respectively. If $(3 m+1) \mu_{1}$ is not less than 2 , then the inequality in Corollary 1.3 is trivial since $\mu_{2} \leq 2$ always holds. In this section, we see that there exist infinitely many graphs such that $(3 m+1) \mu_{1}$ is strictly less than 2 .

Let $C_{N}, N \geq 3$, be the cycle graph with $N$ vertices. Cycle graphs are symmetric. The spectra of cycle graphs are well-known.

Lemma 4.1. The smallest positive eigenvalue of the normalized Laplace operator associated with $C_{N}$ is $1-\cos (2 \pi / N)$ and its multiplicity is 2 .

Since $1-\cos (2 \pi / N)$ is decreasing in $N$ and tends to 0 as $N \rightarrow \infty$, there exists a number $N_{0}$ such that $(3 m+1) \mu_{1}=7(1-\cos (2 \pi / N))$ is strictly less than 2 for any $N \geq N_{0}$. In fact, we can take $N_{0}=9$. 


\section{REFERENCES}

[1] Q.-M. Cheng and H. Yang, Estimates on eigenvalues of Laplacian, Math. Ann. 331 (2005), no. 2, 445-460.

[2] G. N. Hile and M. H. Protter, Inequalities for eigenvalues of the Laplacian, Indiana Univ. Math. J. 29 (1980), no. 4, 523-538.

[3] B. Hua, Y. Lin, and Y. Su, Payne-Pólya-Weinberger, Hile-Protter and Yang's inequalities for Dirichlet Laplace eigenvalues on integer lattices, arXiv:1710.05799 (2017).

[4] L. E. Payne, G. Pólya, and H. F. Weinberger, On the ratio of consecutive eigenvalues, J. Math. and Phys. 35 (1956), 289-298.

[5] L. E. Payne, G. Pólya, and H. F. Weinberger, Sur le quotient de deux fréquences propres consécutives, C. R. Acad. Sci. Paris 241 (1955), 917-919.

[6] C. J. Thompson, On the ratio of consecutive eigenvalues in $N$-dimensions, Studies in Appl. Math. 48 (1969), 281-283.

[7] H. Yang, An estimate of the difference between consecutive eigenvalues, Preprint IC/91/60 of ICTP, Trieste (1995).

Mathematical Institute, Tohoku University, Sendai 980-8578, Japan

E-mail address: shin-ichiro.kobayashi.p3@dc.tohoku.ac.jp 